\title{
School Disengagement of High School Students with Residential Care in Japan
}

Eiji Ozawa1, Yutaro Hirata ${ }^{2}$, and Rumi Fukudome ${ }^{3}$

1 Faculty of Human-Environment Studies, Kyushu University, Japan

${ }^{2}$ Faculty of Law, Economics and the Humanities, Kagoshima University, Japan

${ }^{3}$ Faculty of Human Relations, Fukuoka Jo Gakuin University, Japan

\section{Results}

(3) the period of admitting residential institutions.

We attempt to describe the actual

conditions and educational requirements needed to support for them.

Methods

- The sample consisted of 773 youth in child care institutions in Japan

Youth who were admitted into institutions by 9 th grade, entered high school between 2012 and 2013, and

decided on a course after high school by 2016 were extracted.

2. Measures and Items

- A questionnaire completed by staff of residential institutions for child care.

- Diagnoses of mental and physical

disabilities.

- Types of experience of child abuse and neglect before entering residential institution.

- Placement start date.

-Existence of high school dropout.
1. Characteristics of Study Sample

\begin{tabular}{ccc} 
& $\mathrm{n}$ & $\%$ \\
\hline Total N & 773 & \\
\hline Mender & & \\
\hline Female & 420 & 54.3 \\
\hline & 535 & 45.7 \\
\hline
\end{tabular}

High school outcome

Completion $\quad 624 \quad 80.7$

Dropout $\quad 149 \quad 19.3$

Experience of maltreatment

2. High school dropout rate

$$
0 \% \quad 50 \%
$$

Yes

No

426

55.1

Diagnosis of disabilities

Yes

No

138

44.9

\begin{tabular}{ccc} 
Yes & 138 & 17.9 \\
\hline No & 653 & 82.1 \\
\hline
\end{tabular}

Period of placement

Preschool

293

37.9

Elementary school $\quad 247 \quad 32.0$

Junior high school $\quad 233 \quad 30.1$
Male $20.2 \%$

Female $18.1 \%$

Maltreated $18.5 \%$

None $20.2 \%$

$81.5 \%$

$79.8 \%$

$81.9 \%$

Disabilities $16.7 \% \quad 83.3 \%$

None $19.8 \% \quad 80.2 \%$

Preschool $16.7 \% \quad 83.3 \%$

Elementary school $15.4 \% \quad 84.6 \%$

$\begin{array}{lll}\text { Junior high school } \quad 26.6 \% & 73.4 \%\end{array}$

Dropout Complation
3. Logistic Regression Models of High School Dropout

\begin{tabular}{|c|c|c|}
\hline Variables & $\begin{array}{l}\text { Odds ratio } \\
{[95 \% \mathrm{Cl}]}\end{array}$ & $p$-value \\
\hline \multicolumn{3}{|l|}{ Gender } \\
\hline Female $^{2}$ & $\begin{array}{c}0.83 \\
{[0.58-1.20]}\end{array}$ & .323 \\
\hline
\end{tabular}

Experience of maltreatment

\begin{tabular}{lcc} 
Yes $^{3}$ & 0.89 & .520 \\
& {$[0.61-1.28$} & .520 \\
\hline
\end{tabular}

Diagnosis of disabilities

\begin{tabular}{|c|c|c|}
\hline Yes $^{3}$ & $\begin{array}{c}0.82 \\
{[0.50-1.35]}\end{array}$ & .429 \\
\hline \multicolumn{3}{|l|}{ eriod of placement } \\
\hline Elementary school ${ }^{4}$ & $\begin{array}{c}0.93 \\
{[0.58-1.48]}\end{array}$ & \multirow{2}{*}{.003} \\
\hline Junior high school ${ }^{4}$ & $\begin{array}{c}1.87 \\
{[1.21-2.87]}\end{array}$ & \\
\hline
\end{tabular}

$\mathrm{Cl}=$ confidential interval. ${ }^{1}$ Estimated with Wald's group was "No." " 4 Reference group was "Junio high school."

\section{(20)}

Child maltreatment was not a risk factor for high school dropout.

Youth with disabilities do not suffer a significantly higher risk of high school dropout than those without disabilities.

- The time of admission to residential institutions impacted drop rate risks significantly. Youth admitted at junior high school age had a significantly higher risk of drop out.

Youth receiving short-term care were more likely to have problems in high school adaptation.

The results reveal the importance of educational and psychological support for youth especially those admitted to childcare settings in their adolescence.

\section{Funding:}

This research was funded by JSPS KAKENHI Grant

Acknowledgement:

This work was supported

by the Council of

Children's Home,

Welfare Council in Kyush

area (Kyu-sha-ren Jidou

Yougo Shisetsu Kyougikai) 